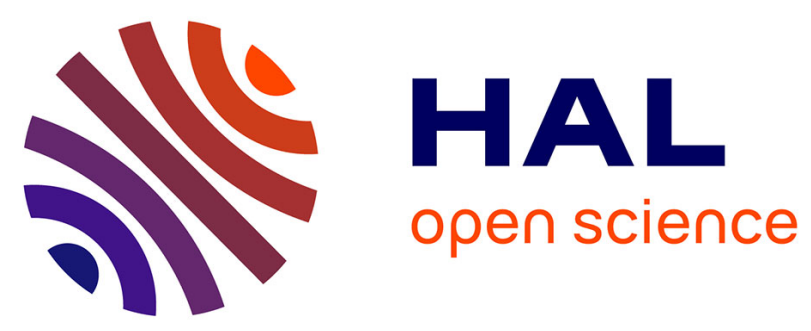

\title{
Collisions between coaxial vortex solitons in the three-dimensional cubic-quintic complex Ginzburg-Landau equation
}

\author{
Dumitru Mihalache, D. Mazilu, F. Lederer, Hervé Leblond, Boris Malomed
}

\section{- To cite this version:}

Dumitru Mihalache, D. Mazilu, F. Lederer, Hervé Leblond, Boris Malomed. Collisions between coaxial vortex solitons in the three-dimensional cubic-quintic complex Ginzburg-Landau equation. Physical Review A : Atomic, molecular, and optical physics [1990-2015], 2008, 77 (3), pp.033817. 10.1103/PhysRevA.77.033817 . hal-03423768

\section{HAL Id: hal-03423768 \\ https://univ-angers.hal.science/hal-03423768}

Submitted on 10 Nov 2021

HAL is a multi-disciplinary open access archive for the deposit and dissemination of scientific research documents, whether they are published or not. The documents may come from teaching and research institutions in France or abroad, or from public or private research centers.
L'archive ouverte pluridisciplinaire HAL, est destinée au dépôt et à la diffusion de documents scientifiques de niveau recherche, publiés ou non, émanant des établissements d'enseignement et de recherche français ou étrangers, des laboratoires publics ou privés. 


\title{
Collisions between coaxial vortex solitons in the three-dimensional cubic-quintic complex Ginzburg-Landau equation
}

\author{
D. Mihalache, ${ }^{1}$ D. Mazilu, ${ }^{1}$ F. Lederer, ${ }^{2}$ H. Leblond, ${ }^{3}$ and B. A. Malomed ${ }^{4}$ \\ ${ }^{1}$ Horia Hulubei National Institute for Physics and Nuclear Engineering (IFIN-HH), \\ 407 Atomistilor, Magurele-Bucharest 077125, Romania \\ ${ }^{2}$ Institute of Solid State Theory and Theoretical Optics, Friedrich-Schiller Universität Jena, Max-Wien-Platz, 1, D-077743 Jena, Germany \\ ${ }^{3}$ Laboratoire POMA, UMR 6136, Université d'Angers, 2 Bd Lavoisier, 49000 Angers, France \\ ${ }^{4}$ Department of Physical Electronics, Faculty of Engineering, Tel Aviv University, Tel Aviv 69978, Israel \\ (Received 20 November 2007; revised manuscript received 23 January 2008; published 7 March 2008)
}

\begin{abstract}
We present generic outcomes of collisions between stable solitons with intrinsic vorticity $S=1$ or $S=2$ in the complex Ginzburg-Landau equation with the cubic-quintic nonlinearity, for the axially symmetric configuration. An essential ingredient of the complex Ginzburg-Landau equation is an effective transverse diffusivity (which is known in models of laser cavities), as vortex solitons cannot be stable without it. For the sake of comparison, results are also included for fundamental three-dimensional solitons, with $S=0$. Depending on the collision momentum, $\chi$, three generic outcomes are identified: merger of the solitons into a single one, at small $\chi$; quasielastic interaction, at large $\chi$; and creation of an extra soliton, in an intermediate region. In addition to the final outcomes, we also highlight noteworthy features of the transient dynamics.
\end{abstract}

DOI: 10.1103/PhysRevA.77.033817

PACS number(s): 42.65.Tg, 42.65.Sf, 47.20.Ky

\section{INTRODUCTION}

The complex Ginzburg-Landau (CGL) equations constitute a mathematical framework for modeling nonlinear dynamics in-situations close to pattern-forming transitions in various dissipative media, such as optical cavities, viscous fluid flows and thermal convection, reaction-diffusion mixtures, and others [1]. An important class of patterns observed in these media and reproduced by the CGL equations are localized (solitary) pulses, alias dissipative solitons (DSs) [2-4]. The search for stable DS solutions is a challenge in modeling two- and three-dimensional (2D and 3D) media, because of the possibility of the critical or supercritical collapse, in the 2D and 3D geometry, respectively, under the action of the self-focusing cubic nonlinearity. In addition to that, multidimensional localized states with intrinsic vorticity, i.e., vortex solitons, are prone to instability against azimuthal perturbations that tend to split them $[5,6]$.

It is well known that a physically relevant model which may support stable DSs in any dimension is provided by the CGL equation with the cubic-quintic (CQ) nonlinearity. This equation was originally introduced by Petviashvili and Sergeev [7] in the 2D form, with the objective to generate vortical DSs. In the 1D version of the same model, solitarypulse solutions and their stability were then investigated in detail [8]. Nonlinear optical media that feature the CQ response include chalcogenide glasses [9] and some organic materials [10].

Actually, stable 2D vortical solitons, with topological charge (vorticity) $S=1$ and 2, were constructed in the framework of the CQ CGL equation in Refs. [11]. Stable fundamental $(S=0)$ 3D localized states were found as spatiotemporal DSs in optical models based on equations of the same type [12-15]. 3D double-soliton complexes, including rotating ones [16], have been found too. Finally, 3D vortex solitons with $S=1,2$, and 3 have been obtained recently, as solutions to the CQ CGL equation $[17,18]$. The stability of the latter solutions was analyzed in terms of the growth rates of perturbation eigenmodes, and verified in direct simulations.

Once stable solitons are available, a problem of great interest is to consider collisions between them. In this work, we aim to investigate collisions in the framework of the following CQ CGL equation in three dimensions $[17,18]$, focusing on vortex solitons:

$$
\begin{aligned}
i U_{z} & +\left(\frac{1}{2}-i \beta\right)\left(U_{x x}+U_{y y}\right)+\left(\frac{D}{2}-i \gamma\right) U_{t t} \\
& +\left[i \delta+(1-i \varepsilon)|U|^{2}-(\nu-i \mu)|U|^{4}\right] U=0 .
\end{aligned}
$$

In terms of nonlinear optics, $U$ is the local amplitude of the electromagnetic wave in the bulk medium which propagates along axis $z$, the transverse coordinates are $x$ and $y$, while the temporal variable is $t=T-z / V_{0}$, where $T$ is time and $V_{0}$ is the group velocity of the carrier wave. The coefficients which are scaled to be $1 / 2$ and 1 account, respectively, for diffraction in the transverse plane and the self-focusing Kerr nonlinearity, $\beta \geq 0$ is the effective diffusivity in the transverse plane, real constants $\delta, \varepsilon$, and $\mu$ represent, respectively, the linear loss, cubic gain, and quintic loss (the basic ingredients of the CQ CGL equation [7]). Here $\nu \geq 0$ accounts for the self-defocusing quintic nonlinearity, that may compete with the cubic term, according to the experimental observations $[9,10], D$ is the group-velocity dispersion (GVD) coefficient $[D>0 \quad(D<0)$ corresponds to the anomalous (normal) GVD], and $\gamma \geq 0$ accounts for the dispersion of the linear loss.

The model to be investigated will be taken in the form of Eq. (1) with zero spectral filtering parameter $(\gamma=0)$, which admits free motion of solitons along axis $z$, and thus makes collisions between them possible [19]. On the contrary to that, free motion in plane $(x, y)$ is impeded by the diffusivity term (the one with $\beta>0$ ) in Eq. (1). As shown in Refs. 


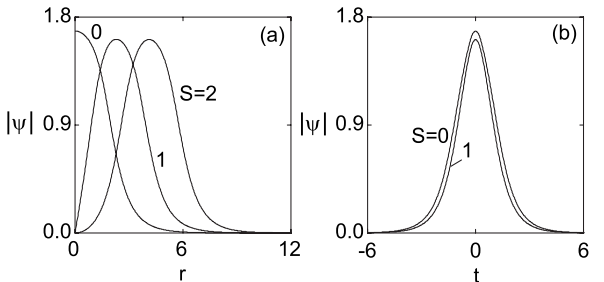

FIG. 1. Cross-section shapes of typical stable 3D dissipative solitons, with $S=0,1,2$, in the transverse $(r)$ and temporal $(t)$ directions, for $\beta=0.5, \mu=1$, and $\varepsilon=2.3$ [in panel (b), the temporal shapes for $S=2$ completely overlap with that for $S=1$ ].

$[17,18]$, this term is necessary for the stability of vortex DSs, while fundamental solitons, with $S=0$, may be stable at $\beta=0$ as well.

In the general case, analysis of collisions between localized objects in the 3D space is quite involved, requiring heavy simulations of the full 3D equations. In particular, in the framework of the CGL equation with $\beta=\gamma=0$, where the free motion is possible both in transverse plane $(x, y)$ and along axis $z$, the collision between zero-spin solitons is controlled by four parameters: relative transverse and axial velocities of the solitons, and two respective aiming parameters [in terms of the optical model, the aiming parameter related to the motion along $z$ is realized as the temporal separation between the solitons when their separation in the $(x, y)$ plane attains a minimum]. Examples of collisions between 3D DSs with $S=0$ were reported in Ref. [15], in the model with $\beta$ $=0$ and $\gamma>0$. Outcomes observed in those simulations included fusion of the solitons into a single one (sometimes, it rather looked as destruction of one of the colliding objects), or quasielastic interaction (mutual bounce of the solitons).

As said above, if the colliding solitons carry vorticity, the CGL equation must include the term proportional to diffusivity parameter $\beta>0$, otherwise the solitons are unstable. Then, as their motion in the transverse plane is suppressed by the diffusivity term, the collision depends on two parameters, $v i z$. , the relative velocity in the axial direction and the related aiming parameter, which is the distance between parallel tra-

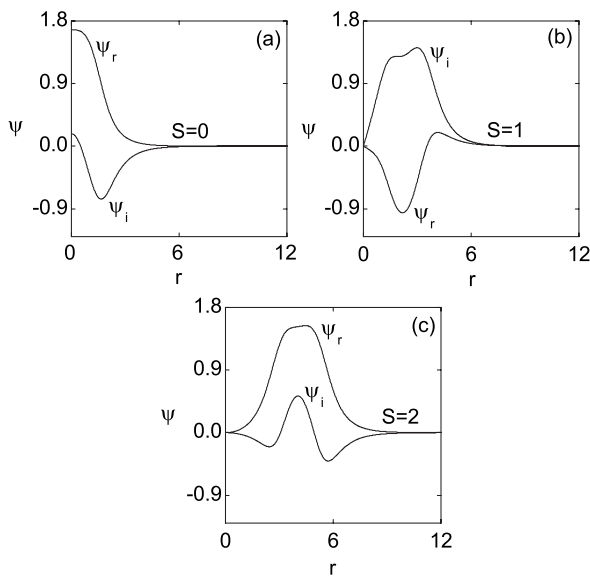

FIG. 2. The real and imaginary parts, $\psi_{\mathrm{r}}$ and $\psi_{\mathrm{i}}$, of the radial shapes for $S=0$ (a), $S=1$ (b), and $S=2$ (c). The parameters are as in Fig. 1.

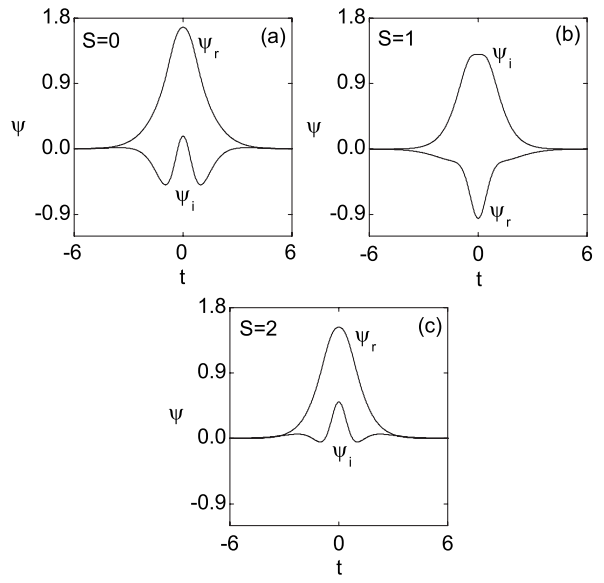

FIG. 3. The real and imaginary parts, $\psi_{\mathrm{r}}$ and $\psi_{\mathrm{i}}$, of the temporal shapes for $S=0$ (a), $S=1$ (b), and $S=2$ (c). The parameters are as in Fig. 1.

jectories of the colliding solitons. In this work, we focus on the more tractable setting, by choosing the diffusivity coefficient $\beta>0$ and zero aiming parameter, when the 3D colliding solitons form a coaxial configuration. A systematic analysis is more feasible in that case, due to the inherent axial symmetry (nevertheless, the numerical integration of the CGL equation is performed in the Cartesian coordinates, which guarantees that all potentially dangerous perturbations are incorporated, including those which may break the axial symmetry). As collisions between 3D vortex solitons have never been studied before, the consideration of the coaxial setting as the first step in tackling this complex problem may be quite appropriate.

The rest of the paper is structured as follows. The model equation and setting for the analysis are formulated in Sec. II, results of the analysis are reported in Sec. III, and the paper is concluded by Sec. IV. The results are formulated in a compact form, which demonstrates the outcome of the collision as a function of parameters of the physical setting.

\section{THE CUBIC-QUINTIC GINZBURG-LANDAU MODEL AND STABLE SOLITONS}

In the general case, the 3D CGL equation with the CQ nonlinearity takes the form of Eq. (1) [17,18]. All coefficients in Eq. (1) are well-known physical parameters, except
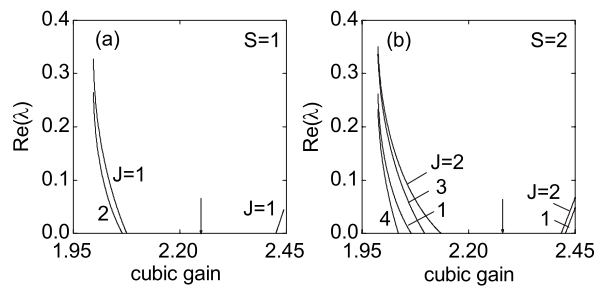

FIG. 4. The largest instability growth rate vs $\varepsilon$ for $3 \mathrm{D}$ vortex dissipative solitons: (a) $S=1$, (b) $S=2$. Other parameters are $\mu=1$, $\beta=0.5$, and $\gamma=0$. The arrows indicate centers of the stability intervals. 


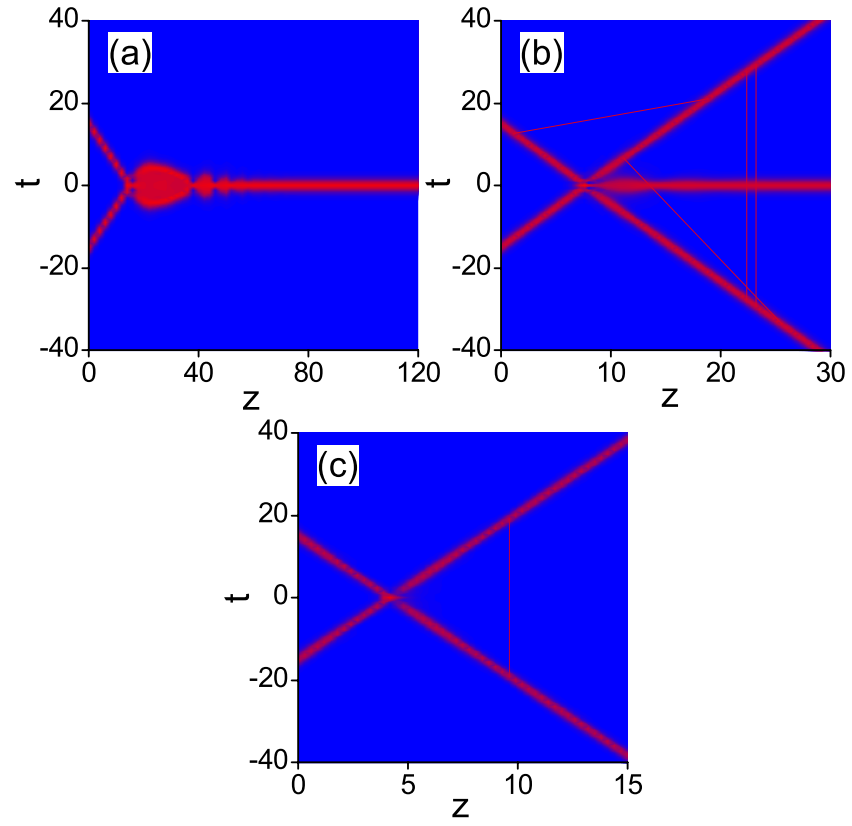

FIG. 5. (Color online). Contour plots display the evolution of field $|U|$ in plane $(t, z)$, for three collision scenarios at different values of kick $\chi$ for the solitons with $S=0$ : (a) merger into a single soliton, at $\chi=1$; (b) creation of an extra soliton, at $\chi=2$; (c) quasielastic collision, at $\chi=4$.

for a more "exotic" one, $\beta$. The diffusivity term proportional to $\beta$ appears in a model of laser cavities, where it is generated by the interplay of the dephasing of the local polarization in the dielectric medium, cavity loss, and detuning between the cavity's and atomic frequencies [20]. As said above, we will keep $\beta>0$, to secure the stability of the vortex solitons, and we will set $\gamma=0$, to admit the free motion of the solitons along axis $z$.

In the conservative counterpart of the CQ CGL equation, i.e., the nonlinear Schrödinger equation with the CQ nonlinearity, the quintic term must be self-defocusing (in the $2 \mathrm{D}$ and 3D geometries), to arrest the collapse induced by the self-focusing cubic term [5,21]. However, the selfdefocusing sign of the quintic term is not necessary for the stability of the multidimensional DSs, because the collapse is prevented by the stronger effect of the quintic term in the dissipative part of the equation [17]. The sign of the GVD coefficient $D$ is not crucial either, because the existence of stable fundamental and vortical 3D solitons was demonstrated for both $D>0$ [17] and $D<0$ [18], both cases being relevant to optics $(D<0$ gives rise to strong phase chirp along the temporal direction in the soliton).

First we look for stationary DS solutions to Eq. (1) in the form of

$$
U(z, x, y, t)=\psi(r, t) \exp (i k z+i S \theta)
$$

where $r$ and $\theta$ are the polar coordinates in plane $(x, y), S$ $\geq 0$ is the above-mentioned integer vorticity (the fundamental solitons correspond to $S=0$ ), $k$ is a real wave number, and complex function $\psi(r, t)$ obeys the stationary equation
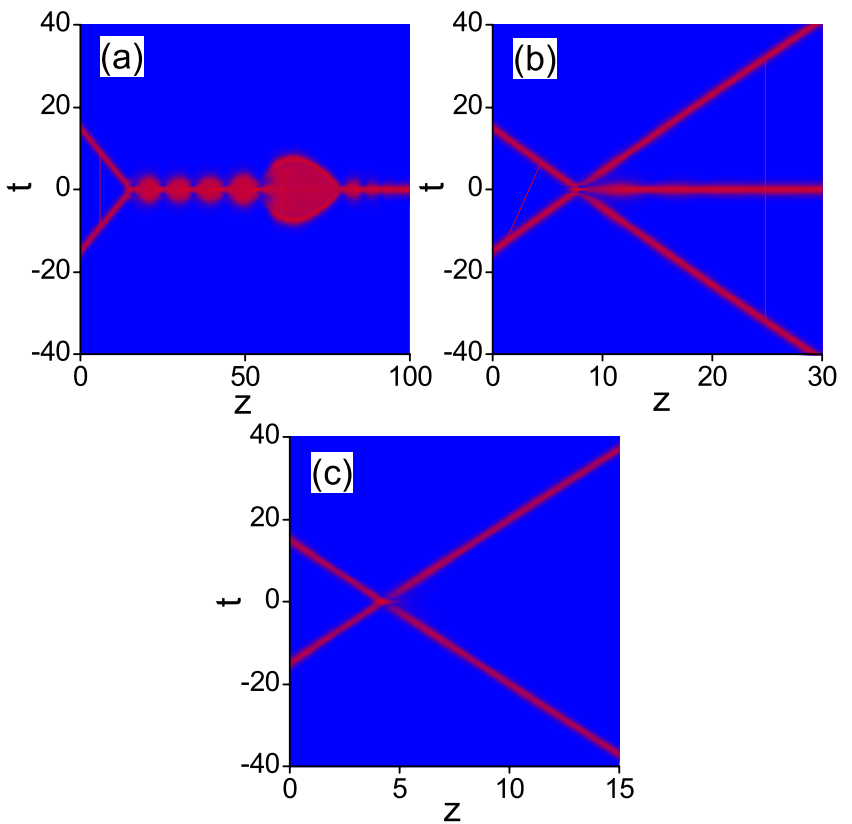

FIG. 6. (Color online). The same as in Fig. 5 but for vortex solitons with $S=1$ : (a) $\chi=1$, (b) $\chi=2$, (c) $\chi=4$.

$$
\begin{aligned}
\left(\frac{1}{2}-i \beta\right)\left(\psi_{r r}+\frac{1}{r} \psi_{r}-\frac{S^{2}}{r^{2}} \psi\right)+\frac{1}{2} D \psi_{t t} \\
+\left[i \delta+(1-i \varepsilon)|\psi|^{2}-(\nu-i \mu)|\psi|^{4}\right] \psi=k \psi,
\end{aligned}
$$

where we set $\gamma=0$, as said above. Localized solutions to this equation must decay exponentially at $r,|t| \rightarrow \infty$, and as $r^{S}$ at $r \rightarrow 0$.

Below, we consider the following set of parameters: $D=1$ (anomalous GVD), $\mu=1, \nu=0.1, \delta=0.4$, and $\beta=0.5$. We will consider stationary DSs with vorticities $S=0, S=1$, and $S=2$. To generate these solutions, we simulated the propagation of zero-vorticity and vortical localized pulses forward in $z$, within the framework of the radial version of Eq. (1), obtained by the substitution of $U(z, x, y, t)$ $=\Psi(z, r, t) \exp (i S \theta)$, using the following axially symmetric input pulses:

$$
\Psi(0, r, t)=A r^{S} \exp \left[-(1 / 2)\left(r^{2} / \rho^{2}+t^{2} / \tau^{2}\right)\right],
$$

with some constants $A, \rho$, and $\tau$. The standard CrankNicholson scheme was used for the numerical integration (typically, with transverse and longitudinal step sizes $\Delta r$ $=\Delta t=0.2$ and $\Delta z=0.01)$. The nonlinear finite-difference equations were solved with the help of the Picard iteration method, and the resulting linear system was then handled by means of the Gauss-Seidel iterative procedure. To achieve reliable convergence to stationary states, ten Picard and four Gauss-Seidel iterations were usually sufficient. The wave number $(k)$ of an established solution was found as the corresponding value of the $z$ derivative of the phase of $\Psi(z, r, t)$, provided that it became independent on $z, r$, and $t$ up to five significant digits. Typical radial and temporal cross sections of the stable DSs with $S=0,1$ and $S=2$ are shown in Figs. 1-3 for $\epsilon=2.3$ [the radial and temporal shapes are shown, respectively, across $t=0$ and $r=r_{\max }$, where local power 


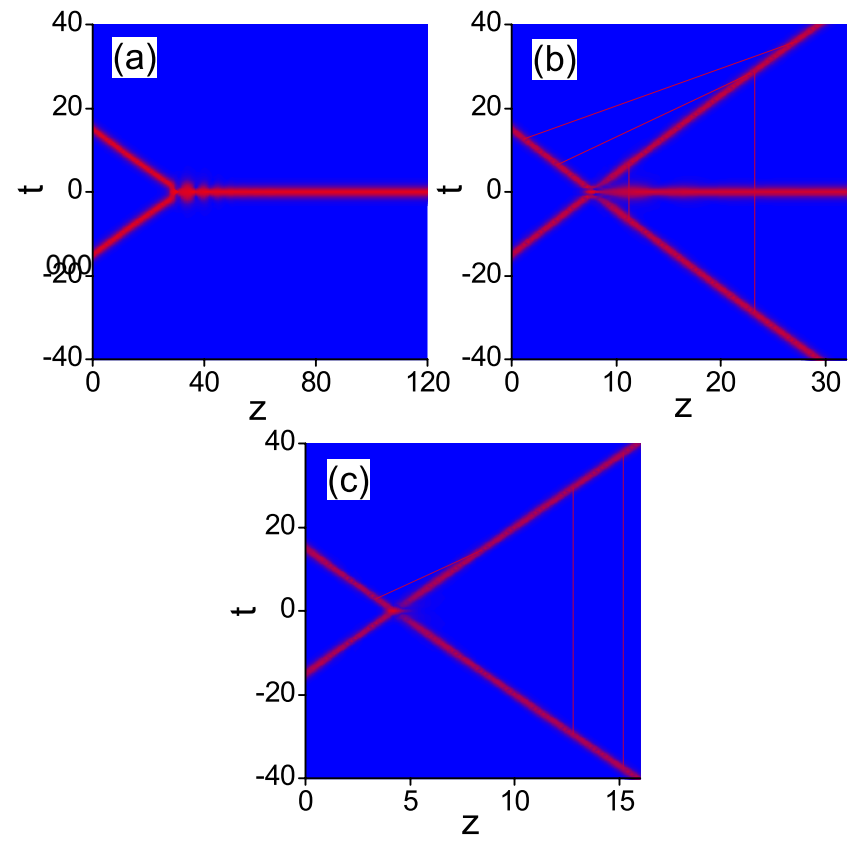

FIG. 7. (Color online). The same as in Fig. 5, but for vortex solitons with $S=2$ : (a) $\chi=0.5$, (b) $\chi=2$, (c) $\chi=4$.

$|\psi(r, t)|^{2}$ attains its maximum]. It is noteworthy that, while the temporal shapes of the solitons with $S=1$ and 2 are virtually identical in terms of $|\psi|$, they are quite different as concerns their real and imaginary parts; see Figs. 3(b) and 3 (c). The wave numbers (with five significant digits) corresponding to the DSs shown in Fig. 1 are $k=0.44342$ for $S$ $=0, k=0.50040$ for $S=1$, and $k=0.50387$ for $S=2$.

We stress that the above numerical procedure does not guarantee the stability of the so found vortex states, as they may be subject to instability against azimuthal perturbations $[17,18]$. The full stability of the vortex DSs was identified through the computation of instability growth rates for eigenmodes of small perturbations. To this end, a perturbed solution to Eq. (1) was looked for as

$$
\begin{aligned}
U= & {\left[\psi(r, t)+f(r, t) \exp (\lambda z+i J \theta)+g^{*}(r, t) \exp \left(\lambda^{*} z-i J \theta\right)\right] } \\
& \times \exp (i k z+i S \theta),
\end{aligned}
$$

where integer $J$ and (generally, complex) $\lambda$ are the azimuthal index and growth rate of infinitesimal perturbations represented by eigenmodes $f$ and $g$. The substitution of this expression in Eq. (1) leads to linearized equations which were
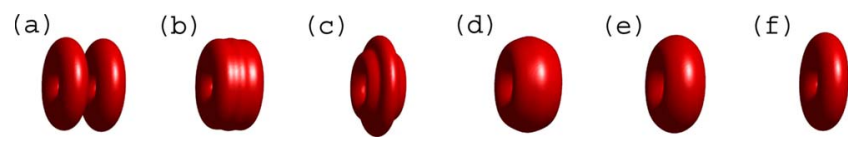

FIG. 8. (Color online). Isosurface plots of total intensity $|U(x, y, t)|^{2}$, showing the precollision and postcollision dynamics in the case of the merger, for different values of the propagation distance: $z=25$ (a), $z=28$ (b), $z=30$ (c), $z=35$ (d), $z=40$ (e), $z=60$ (f). Here $S=2, \chi=0.5$, and the simulations were run on the grid of size $201 \times 201 \times 501$.
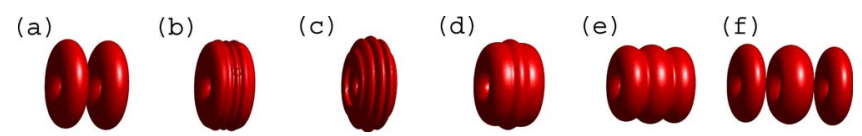

FIG. 9. (Color online). The same as in Fig. 8, in the case of the creation of an extra soliton, for $S=2$ and $\chi=2$. Here, $z=6$ (a), $z$ $=7$ (b), $z=8$ (c), $z=9$ (d), $z=10$ (e), $z=12$ (f). The simulations were run on the grid of size $201 \times 201 \times 501$.

solved by means of the same numerical methods as in Refs. $[17,18]$.

The fundamental DSs are stable in their entire existence domain, whereas the vortex solitons are stable only in a part of their existence domain $[17,18]$. For our choice of the parameters we have found that the solitons are stable in the following intervals of variation of the cubic gain parameter $\varepsilon: 2.144 \leq \varepsilon \leq 2.373$ (for $S=0$ ), $2.075 \leq \varepsilon \leq 2.425$ (for $S=1$ ), $2.135 \leq \varepsilon \leq 2.417$ (for $S=2$ ). These intervals of stability are outcomes of the linear stability analysis for the vortex DSs, see Fig. 4, where, fixing $\mu=1$, we display the largest instability growth rate vs nonlinear gain $\varepsilon$ for vortex dissipative solitons with $S=1$ and $S=2$. Note that actual borders of the stability regions for the vortex solitons with vorticity $S$ are determined by the perturbation eigenmodes with $J=S$.

Thus to study generic outcomes of collisions between DSs, one should take a pair of stable solitons with shapes shown above, separated by large temporal distance $T$. The solitons are set in motion by "kicking" them in the axial direction, i.e., multiplying each soliton by $\exp ( \pm i \chi t)$. Note that, with $\gamma=0$, Eq. (1) supports the Galilean invariance in this direction, i.e., the application of the kick to the original soliton, $U_{0}(z, t, x, y)$, generates an exact solution in the form of a "walking" soliton, $U_{\chi}(z, t, x, y)$ $=U_{0}(z, t \mp a \chi, x, y) \exp \left( \pm i \chi t-i a \chi^{2} z / 2\right)$.

\section{RESULTS}

To solve Eq. (1) with the initial condition (at $z=0$ ) corresponding to collisions between two stable identical solitons, initially separated by large temporal distance $T$, a fully 3D implicit (Crank-Nicholson) finite-difference scheme was used, with typical transverse and longitudinal step sizes $\Delta x$ $=\Delta y=\Delta t=0.2$ and $\Delta z=0.01$. Similar to the simulations outlined in the previous section, the resulting nonlinear finitedifference equations were solved using the Picard iteration method, and the ensuing linear system was then dealt using the Gauss-Seidel elimination procedure. To achieve good convergence, ten Picard and four Gauss-Seidel iterations were typically needed. In most cases, we used 501 discreti-

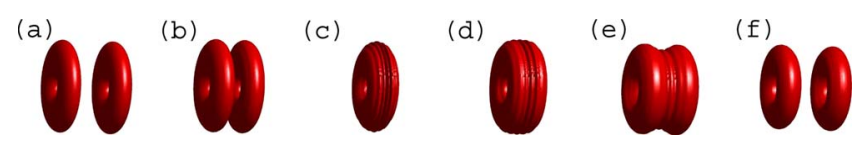

FIG. 10. (Color online). The same as in Fig. 8 in the case of a quasielastic collision, for $S=2$ and $\chi=4$. Here, $z=3$ (a), $z=3.5$ (b), $z=4$ (c), $z=4.5$ (d), $z=5$ (e), $z=5.5$ (f). The simulations were run on the grid of size $201 \times 201 \times 501$. 

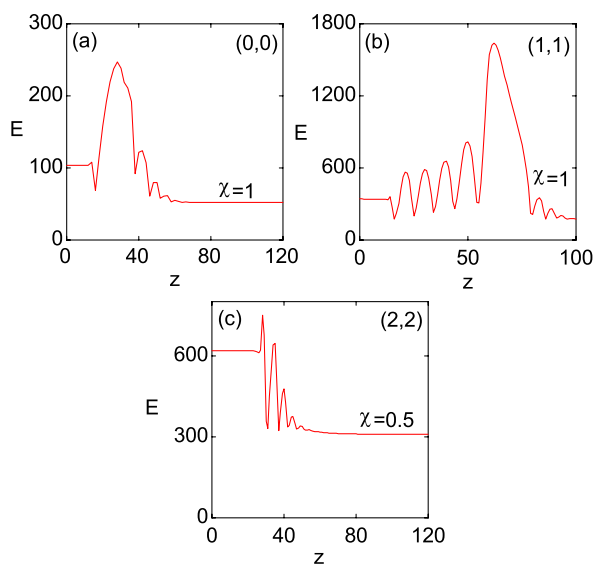

FIG. 11. (Color online). The evolution of the total energy in the case of the merger into a single soliton, for $S=0, \chi=1$ (a); $S=1$, $\chi=1$ (b); $S=2, \chi=0.5$ (c).

zation points for $t$, while the number of the mesh points for $x$ and $y$ that provided for the required accuracy depended on the vorticity: $160 \times 160$ points for $S=0,193 \times 193$ for $S=1$, and $201 \times 201$ for $S=2$.

Results of systematic simulations of soliton collisions may be adequately represented by outcomes observed at the following values of parameters in Eq. (1): $D=1$ (anomalous GVD), $\mu=1, \varepsilon=2.3, \nu=0.1, \delta=0.4$, and $\beta=0.5$ (recall that we also set $\gamma=0$ ), while kick parameter $\chi$ was varied. In this case, the 3D solitons with $S=0,1,2$ (and also with $S=3$ ) are all stable $[17,18]$, being characterized by the following values of the energy (alias norm):

$$
E \equiv 2 \pi \int_{0}^{\infty} r d r \int_{-\infty}^{+\infty} d t|\psi(r, t)|^{2}
$$

$E(S=0) \approx 52, E(S=1) \approx 171$, and $E(S=2) \approx 310$.

We have studied collisions for DS pairs with vorticities $(S, S)=(0,0),(1,1)$, and $(2,2)$. While we are chiefly interested in the interactions between vortex solitons, the results for $S$ $=0$ are included too, for comparison.

Gradually increasing initial kick $\chi$, we have observed the following outcomes (the initial separation was typically $T$ $=30$, but variation of $T$ did not affect the results):

(i) Merger of the two solitons into one, at small values of $\chi$, namely, in intervals $\chi \leq 1.1$ for $S=0, \chi \leq 1.2$ for $S=1$, and $\chi \leq 1.4$ for $S=2$.

(ii) Generation of an extra soliton, according to scheme $1+1 \rightarrow 1+1+1$, at intermediate values of $\chi$, namely, in intervals $1.1<\chi \leq 2.2$ for $S=0,1.2<\chi \leq 2.4$ for $S=1$, and 1.4 $<\chi \leq 2.4$ for $S=2$. Note that a similar effect of "soliton birth" was observed experimentally in the case of collinear collisions of two one-dimensional dissipative spatial solitons in periodically patterned semiconductor amplifiers [22]. Our results show that the soliton creation is a generic feature of collisions of both fundamental $(S=0)$ and spinning $(S \neq 0)$ solitons described by the CGL equation.
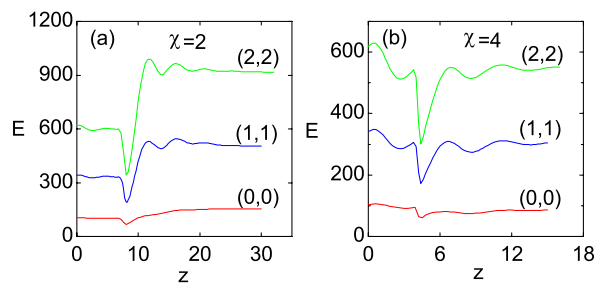

FIG. 12. (Color online). The same as in Fig. 11: (a) when the collision results in the creation of an extra soliton $(\chi=2)$; (b) for the quasielastic collision $(\chi=4)$.

(iii) Quasielastic interactions at larger $\chi$, i.e., $\chi>2.2$ for $S=0, \chi>2.4$ for $S=1$, and $\chi>2.4$ for $S=2$. In this case, the solitons pass through each other, and after the collision they feature velocities slightly smaller than they had originally.

These three collision scenarios are illustrated in Figs. 5-7 by pictures of the evolution of the field in the plane of $(t, z)$.

Another detailed illustration of the three generic outcomes of the collisions (for the solitons with $S=2$ ) is provided by the 3D plots displayed in Figs. 8-10. These figures clearly show that the solitons suffer considerable deformations in the course of the collisions. Also the total energy (norm) of the solitons strongly changes during the collision process. The evolution of the total energy is shown in Fig. 11 (for the case of the merger) and Fig. 12 (for the cases of the creation of the extra soliton, and quasielastic collision). In these figures, labels $(0,0),(1,1)$, and $(2,2)$ stand for the respective values of $S$ of the colliding solitons.

In the case shown in Fig. 11, the merger is signalized by eventual drop of the energy to half of the initial level, while the appearance of the extra soliton is seen through the jump of the energy to $150 \%$ of the initial value. In the case of the quasielastic collision, the energy drops temporarily, but eventually recovers the initial value [the curves for $S=2$ and $S=1$ in Fig. 12(b) need additional propagation distance to stabilize at the original energy levels].

Note that the energy may attain very high values at an intermediate stage of the merger [exceeding the initial energy by a factor of $\simeq 5$ in Fig. 11(b)], before dropping eventually to $50 \%$ of the initial value. On the contrary, in the case of the generation of the extra soliton, the total energy may temporarily drop to $\simeq 50 \%$ of the initial value, before establishing itself at the eventual level of $150 \%$ [see the curve for $S=2$ in Fig. 12(a)].

\section{CONCLUSION}

We have performed a systematic analysis of collisions between stable 3D dissipative solitons with vorticities $S=1$ and 2, as well as $S=0$, in the complex Ginzburg-Landau equation with the cubic-quintic nonlinearity. The simulations were carried out for the axially symmetric configuration, but using the Cartesian coordinates, hence we may be sure that the entire dynamical process is stable against symmetrybreaking perturbations. Depending on the initial kick applied to the solitons, $\pm \chi$, three generic outcomes of the collisions have been identified, for all values of $S$ : merger of the solitons into a single one, at small $\chi$; quasielastic passage 
through each other, at large $\chi$; and the generation of an extra soliton, at intermediate values of $\chi$ (the process of "soliton birth"). Not only the outcomes, but also transient dynamics have been studied in detail.

This paper reports results of the first analysis of collisions between vortex solitons in the $3 \mathrm{D}$ space. The analysis may be extended in various directions, including on-axis collisions between two solitons with different vorticities (in par- ticular, one of them may have $S=0$ ), and noncoaxial collisions in a more general geometric setting.

\section{ACKNOWLEDGMENT}

This work was supported, in part, by the Deutsche Forschungsgemeinschaft (DFG), Bonn.
[1] I. S. Aranson and L. Kramer, Rev. Mod. Phys. 74, 99 (2002); P. Mandel and M. Tlidi, J. Opt. B: Quantum Semiclassical Opt. 6, R60 (2004); B. A. Malomed, in Encyclopedia of Nonlinear Science, edited by A. Scott (Routledge, New York, 2005), p. 157.

[2] Dissipative Solitons, Lecture Notes in Physics No. 661, edited by N. Akhmediev and A. Ankiewicz (Springer, Berlin, 2005).

[3] S. Barland, J. R. Tredicce, M. Brambilla, L. A. Lugiato, S. Balle, M. Giudici, T. Maggipinto, L. Spinelli, G. Tissoni, T. Knodl, M. Miller, and R. Jager, Nature (London) 419, 699 (2002); Z. Bakonyi, D. Michaelis, U. Peschel, G. Onishchukov, and F. Lederer, J. Opt. Soc. Am. B 19, 487 (2002); E. A. Ultanir, G. I. Stegeman, D. Michaelis, C. H. Lange, and F. Lederer, Phys. Rev. Lett. 90, 253903 (2003); N. N. Rosanov, S. V. Fedorov, and A. N. Shatsev, ibid. 95, 053903 (2005).

[4] N. N. Rosanov, Spatial Hysteresis and Optical Patterns (Springer, Berlin, 2002).

[5] B. A. Malomed, D. Mihalache, F. Wise, and L. Torner, J. Opt. B: Quantum Semiclassical Opt. 7, R53 (2005).

[6] A. S. Desyatnikov, Y. S. Kivshar, and L. Torner, Prog. Opt. 47, 291 (2005).

[7] V. I. Petviashvili and A. M. Sergeev, Dokl. Akad. Nauk SSSR 276, 1380 (1984) [Sov. Phys. Dokl. 29, 493 (1984)].

[8] B. A. Malomed, Physica D 29, 155 (1987); O. Thual and S. Fauve, J. Phys. (Paris) 49, 1829 (1988); S. Fauve and O. Thual, Phys. Rev. Lett. 64, 282 (1990); W. van Saarloos and P. C. Hohenberg, ibid. 64, 749 (1990); V. Hakim, P. Jakobsen, and Y. Pomeau, Europhys. Lett. 11, 19 (1990); B. A. Malomed and A. A. Nepomnyashchy, Phys. Rev. A 42, 6009 (1990); P. Marcq, H. Chaté, and R. Conte, Physica D 73, 305 (1994); N. Akhmediev and V. V. Afanasjev, Phys. Rev. Lett. 75, 2320 (1995); H. R. Brand and R. J. Deissler, ibid. 63, 2801 (1989); R. J. Deissler and H. R. Brand, ibid. 72, 478 (1994); 74, 4847 (1995); 81, 3856 (1998); V. V. Afanasjev, N. Akhmediev, and J. M. Soto-Crespo, Phys. Rev. E 53, 1931 (1996); J. M. Soto-
Crespo, N. Akhmediev, and A. Ankiewicz, Phys. Rev. Lett. 85, 2937 (2000).

[9] F. Smektala, C. Quemard, V. Couderc, and A. Barthélémy, J. Non-Cryst. Solids 274, 232 (2000); G. Boudebs, S. Cherukulappurath, H. Leblond, J. Troles, F. Smektala, and F. Sanchez, Opt. Commun. 219, 427 (2003).

[10] C. Zhan, D. Zhang, D. Zhu, D. Wang, Y. Li, D. Li, Z. Lu, L. Zhao, and Y. Nie, J. Opt. Soc. Am. B 19, 369 (2002).

[11] L.-C. Crasovan, B. A. Malomed, and D. Mihalache, Phys. Rev. E 63, 016605 (2000); Phys. Lett. A 289, 59 (2001).

[12] P. Grelu, J. M. Soto-Crespo, and N. Akhmediev, Opt. Express 13, 9352 (2005).

[13] J. M. Soto-Crespo, P. Grelu, and N. Akhmediev, Opt. Express 14, 4013 (2006).

[14] V. Skarka and N. B. Aleksić, Phys. Rev. Lett. 96, 013903 (2006).

[15] N. Akhmediev, J. M. Soto-Crespo, and P. Grelu, Chaos 17, 037112 (2007).

[16] J. M. Soto-Crespo, N. Akhmediev, and P. Grelu, Phys. Rev. E 74, 046612 (2006).

[17] D. Mihalache, D. Mazilu, F. Lederer, Y. V. Kartashov, L.-C. Crasovan, L. Torner, and B. A. Malomed, Phys. Rev. Lett. 97, 073904 (2006).

[18] D. Mihalache, D. Mazilu, F. Lederer, H. Leblond, and B. A. Malomed, Phys. Rev. A 75, 033811 (2007); 76, 045803 (2007).

[19] H. Sakaguchi, Physica D 210, 138 (2005).

[20] J. Lega, J. V. Moloney, and A. C. Newell, Phys. Rev. Lett. 73, 2978 (1994); Physica D 83, 478 (1995).

[21] D. Mihalache, D. Mazilu, L. C. Crasovan, I. Towers, A. V. Buryak, B. A. Malomed, L. Torner, J. P. Torres, and F. Lederer, Phys. Rev. Lett. 88, 073902 (2002).

[22] E. A. Ultanir, G. I. Stegeman, C. H. Lange, and F. Lederer, Opt. Lett. 29, 283 (2004). 